\title{
The Effects of Muscle Fatigue on Neuromuscular Function and Anterior Tibial Translation in Healthy Knees*
}

\author{
Edward M. Wojtys, † MD, Bradford B. Wylie, and Laura J. Huston, MS \\ From MedSport, the Section of Orthopaedic Surgery, University of Michigan, \\ Ann Arbor, Michigan
}

\begin{abstract}
We investigated the effect of quadriceps and hamstring muscle fatigue on anterior tibial translation and muscle reaction time in 10 healthy subjects. The six men and four women had an average age of 21.3 years and had no known pathologic knee conditions. Each patient underwent a knee examination, arthrometer measurements of tibial translation, subjective functional assessment, and an anterior tibial translation stress test before and after quadriceps and hamstring musclefatiguing exercise. The recruitment order of the lower extremity muscles in response to anterior tibial translation did not change with muscle fatigue. However, the results showed an average increase of $32.5 \%$ in anterior tibial translation (range, $11.4 \%$ to $85.2 \%$ ) after fatigue. Muscle responses in the gastrocnemius, hamstring, and quadriceps originating at the spinal cord and cortical level showed significant slowing and, in some cases, an absence of activity after the quadriceps and hamstring muscles were fatigued. The increases in displacement after fatigue strongly correlated $(0.62$ to 0.96$)$ with a delay in cortical-level activity (intermediate and voluntary). Muscle fatigue, which appears to affect the dynamic stability of the knee, alters the neuromuscular response to anterior tibial translation. Therefore, fatigue may play an important role in the pathomechanics of knee injuries in physically demanding sports.
\end{abstract}

Knee stability depends on both passive and active restraints to tibiofemoral motion. Previous studies by Grana

\footnotetext{
* Presented at the meeting of the AOSSM/Japanese Orthopaedic Society for Sports Medicine Trans-Pacific, Maui, Hawaii, March 1993.

$\dagger$ Address correspondence and reprint requests to Edward $M$. Wojtys, MD, MedSport, POB 363, Ann Arbor, MI 48106.

No author or related institution has received any financial benefit from research in this study.
}

and Muse, ${ }^{9}$ Skinner et al., ${ }^{16}$ Steiner et al., ${ }^{17}$ Stoller et al., ${ }^{18}$ and Weisman et al. ${ }^{20}$ have demonstrated increases in knee laxity after exercise. With presumed muscle relaxation during testing, these laxity changes were attributed to the passive restraint system of the knee because the collagenous components of the knee joint ligaments and capsule were expected to show the typical viscoelastic changes seen in cyclic loading. ${ }^{4,23}$ However, the effect of muscle fatigue on knee joint laxity remains unclear. The goal of this investigation was to test the null hypothesis that fatigue of the quadriceps and hamstring muscles does not affect 1) anterior tibial translation; 2) muscle reaction time in the quadriceps, hamstring, and gastrocnemius muscles; and 3) muscle recruitment order. A secondary goal was to determine whether the changes seen in anterior tibial translation could be traced to the active or passive restraint system.

Presently, the relationship between knee joint laxity, muscle fatigue, and knee joint injury remains unknown. Intuitively, it seems likely that the dynamic restraint system of the knee can prevent or lessen injury during rigorous physical activity. Goldfuss et al. ${ }^{8}$ demonstrated the importance of a quadriceps muscle contraction in stiffening the medial side of the knee in vitro, reporting a $48 \%$ increase in stiffness with quadriceps muscle activation. Wang and Walker ${ }^{19}$ demonstrated the ability of the hamstring and quadriceps muscles to decrease internal and external rotation of the tibia by $80 \%$ with the equivalent of 1 body weight of compressive force. In vivo studies in athletes have demonstrated a tenfold increase in knee stiffness with muscle contraction. ${ }^{13}$

Lower extremity muscle fatigue during rigorous exercise or physical labor may decrease the potential of the dynamic knee defense mechanisms. Indirect evidence of this problem is seen when muscle fatigue adversely affects the spatial-temporal aspects of fine motor control. This is exemplified by a tired basketball player attempting a jump shot. This athlete's form and timing will probably not be as good as when he began the game. The key 
question for the athlete becomes whether or not spinal reflexes or cortical protective responses can be effective in providing dynamic knee stability despite decreasing torque output of the muscle-tendon units as they fatigue. If these neuromuscular elements of the knee joint protection are impaired during strenuous exercise, then attempts to better condition the athlete's dynamic restraint system may be warranted. Unfortunately, it is unclear at this time whether proprioceptive endurance training aimed at a tibial control reflex response or a progressive resistance muscle conditioning program should be pursued when training athletes at risk for knee injury. Traditionally, muscle strength has been emphasized to prevent joint injury in demanding contact sports. Although numerous strength parameters are available for testing, there is little doubt that superior strength alone will not eliminate the risk of knee joint injury. The speed at which the available muscle strength can be generated may play an even greater role in injury prevention. Unfortunately, our abilities to test these facets of neuromuscular control are limited currently. Despite these shortcomings, muscle reaction time seems a worthwhile parameter to investigate and attempt to improve before athletic participation in rigorous and contact sports.

\section{MATERIALS AND METHODS}

\section{Subjects}

We recruited 10 subjects who had no known pathologic knee conditions (six men and four women; average age, 21.3 years; range, 20 to 24). The activity level of each participant was graded based on the intensity of the subject's lower extremity exercise (Table 1). Varied levels of activities among the participants were expected.

\section{Knee Testing Apparatus}

The testing apparatus was designed to measure anterior tibial translation in reference to the femur in response to an anteriorly directed 30-pound step force applied to the posterior aspect of the proximal leg (see Fig. 1, p. 189, March/April 1996). Lower extremity muscle function was recorded simultaneously using surface EMG at five muscle locations (medial and lateral quadriceps, medial and lateral hamstring, and gastrocnemius). The induced anterior tibial translation was used to stimulate spinal cord reflex and voluntary muscle activity that were then ana-

TABLE 1

Physical Activity Level Scale

\begin{aligned} & \hline Level \multicolumn{1}{c}{ Activity } \\ & \hline 10 Competitive jumping, turning, twisting sports \\ & 8 Recreational jumping, turning, twisting sports \\ & 6 Jog, bike, swim, occasional pivoting sports \\ & 4 No jumping, turning, twisting sports; swim, bike, \\ & jog regularly \\ & 2 No jumping, turning, twisting sports; occasionally \\ & jog, swim, bike \\ & 0 Inactive, sedentary \\ & \hline\end{aligned}

lyzed. The accuracy and reproducibility of this anterior tibial translation system have previously been reported. ${ }^{11,21}$

During testing, the subjects were comfortably positioned with their knees maintained at $30^{\circ}$ of flexion by adjustment of an ischial support specifically designed to allow uninhibited hamstring muscle activity. The foot was fixed with the ankle at $10^{\circ}$ to $15^{\circ}$ of dorsiflexion on a bathroom scale to monitor weightbearing status. Weightbearing was maintained in the 20 - to 30 -pound range. Tibial translations, rotations, and subluxations were not restrained in any way. Two linear potentiometers were used to measure anterior tibial translation. Relative tibial displacement was quantified by placing one potentiometer on the patella with the second potentiometer placed on the tibial tuberosity.

\section{Electromyographic Recordings}

Surface EMG recordings were taken from five muscle locations: the lateral quadriceps, medial quadriceps, lateral hamstring, medial hamstring, and the gastrocnemius muscles. Bipolar surface electrodes (Hewlett Packard, Waltham, Massachusetts) were consistently placed over the midregion of each muscle group oriented along the muscle belly. Bipolar electrodes were spaced $3 \mathrm{~cm}$ apart. Before electrode placement, the skin was prepared with sandpaper and cleaned with isopropyl alcohol to assure adequate surface contact. The raw EMG signal was amplified at $100 \mu \mathrm{V} / \mathrm{V}$ and fed into a Zenith Data Systems 286 computer (Zenith Data Systems, St. Joseph, Michigan) for storage and data analysis. The EMG recordings were sampled at a frequency rate of $1000 \mathrm{~Hz}$ over 2.5 seconds, beginning 0.5 seconds before the onset of the anteriorly directed tibial force. The displacing forces were applied at random intervals ( 0.1 to 10 seconds) to prevent anticipatory efforts by the participant. All visual and audio clues associated with the displacing force were eliminated.

\section{Testing Protocol-Anterior Tibial Translation Stress Test}

All subjects underwent two testing sessions; a baseline test was conducted before muscle-fatiguing exercise and a second test was done immediately after quadriceps and hamstring muscle fatigue was reached. The first testing session determined the baseline gastrocnemius, hamstring, and quadriceps muscle reaction times and the anterior tibial translation with muscles relaxed and responding. The second testing session measured the same variables immediately after the hamstring and quadriceps muscles were fatigued on an isokinetic dynamometer. Fatigue was defined as a greater than $50 \%$ decrease in work output (in foot-pounds) in both the quadriceps and hamstring muscle groups.

The dominant lower extremity (dominance was determined by the dominant hand) was always tested first. After a brief orientation during which the anteriorly directed step force was applied to the extremity several times, two types of tests were performed. A relaxed test 
was performed by asking participants to relax their muscles while the force was applied. If muscle activity was detected on EMG at the start of the test, the data were not used, and the test was repeated. A response test was performed by asking the subjects to resist the anterior force after the onset of the anteriorly directed force was detected. Any trial in which the subject contracted the lower extremity musculature before the onset of the anteriorly directed force was discarded and then repeated. Ten trials of each test were recorded and subsequently averaged.

\section{Strength Testing}

After recording baseline EMG activity and anterior tibial translation values with the knee testing apparatus, baseline quadriceps and hamstring muscle strengths (peak torque [in foot-pounds]/body weight [in pounds] $\times 100$ ) were measured on a dynamometer using isokinetic, concentric knee flexion and extension exercises at 60 and 240 $\mathrm{deg} / \mathrm{sec}$. No attempt was made to exercise the gastrocnemius muscle. Throughout all strength testing, all subjects received constant verbal support to encourage consistent maximum effort.

After baseline strength levels were established, the dominant leg was fatigued on the isokinetic dynamometer at $240 \mathrm{deg} / \mathrm{sec}$ for 85 to 135 repetitions, depending on the subject's ability. Fatigue was defined as a greater than $50 \%$ decrease in work (in foot-pounds) from the first five repetitions to the last five repetitions performed in the series for both the quadriceps and hamstring muscle groups. The number of repetitions to be performed was estimated before the fatiguing exercise. Previous experience has shown that the number of repetitions necessary to induce fatigue could be determined sufficiently from the subject's activity category. Subjects in activity category 10 required approximately 135 repetitions; category 9, 120; and categories 7 to 8,110 . This method was successful in keeping each subject's percentage of fatigue in a consistent range $(50 \%)$ for purposes of this study.

To ensure participant safety and to minimize muscle recovery after fatiguing exercise and before muscle reaction time and anterior tibial translation testing, a wheelchair was used to transport the participants from the isokinetic dynamometer to the knee testing device immediately after fatigue induction. Once the participant was placed in the testing device, the EMG leads were attached and the linear potentiometers were positioned identically to the positions used during baseline testing of the muscle. Two muscle response trials were then performed and EMG recordings and anterior tibial translation measurements were obtained. The elapsed time between the completion of the isokinetic exercise and the completion of the two muscles-responding trials was less than 100 seconds for all participants. To determine the effect of muscle fatigue on anterior tibial translation and muscle reaction time, the two response trials were averaged and compared with baseline values.

\section{Data Analysis}

Muscle reaction times were determined by measuring the time span between the onset of the 30-pound displacement force and the first occurrence of muscle activity. The three regions of the EMG signal that were analyzed were the spinal reflex and the intermediate and voluntary muscle activity. Movement artifact was identified by pattern recognition and eliminated before the data analysis.

For each subject and trial, the timing of the spinal reflex, intermediate activity, and voluntary activity was recorded for each muscle group. The 10 spinal reflex times for each muscle group were used to obtain an average spinal reflex time for each of the muscle groups tested. The same calculations were done for intermediate and voluntary activity. The anterior tibial translation results for the 10 baseline response trials were also averaged. The same procedure was used in the fatigue-response trials to obtain average values for muscle reaction time and anterior tibial translation after muscle fatigue. These values were then compared with the baseline values. The average values for muscle reaction time and anterior tibial translation for the 10 subjects were also calculated.

Although not statistically significant, it was noted that the anterior tibial translation values for the muscles-relaxed test were consistently lower during the first three to four trials of the initial series, compared with the remaining and subsequent tests. Therefore, a series of five to seven "pretrials" was added to the protocol to allow patients to become acclimated to the testing device and to allow their anterior tibial translation to reach equilibrium.

During statistical analysis, it became evident that there was occasionally insufficient muscle response data for a separate analysis of the medial and lateral quadriceps muscles. Consequently, the medial and lateral quadriceps muscles were grouped together as simply the quadriceps muscle for statistical significance calculations.

Correlations between muscle strength and activity level were calculated using a matrix of Pearson correlation coefficients. All other comparisons were calculated using paired, two-tailed Student's $t$-tests. All significance was determined at a level of $P \leq 0.05$, but was adjusted for multiple comparisons.

\section{RESULTS}

\section{Activity Level}

The average subjective activity category for the study was 6.2 (range, 2 to 10 ).

\section{Displacements}

Baseline. The average anterior tibial translation in the muscles-relaxed test was $4.25 \mathrm{~mm}$ (range, 1.71 to 8.93 ) (Table 2). The average anterior tibial translation in the muscles-responding test was $2.08 \mathrm{~mm}$ (range, 0.60 to 4.66).

Postfatigue. After muscle fatigue, all subjects displayed an increase in average anterior tibial translation com- 
TABLE 2

Average Change in Anterior Tibial Translation from Baseline to Postfatigue (in milliseconds)

\begin{tabular}{|c|c|c|c|c|c|c|c|c|c|c|}
\hline \multirow{4}{*}{ Patient } & \multicolumn{10}{|c|}{ Groups } \\
\hline & \multicolumn{6}{|c|}{ Baseline } & \multicolumn{4}{|c|}{ Postfatigue } \\
\hline & \multicolumn{3}{|c|}{ Muscles relaxed } & \multicolumn{3}{|c|}{ Muscles respond } & \multicolumn{3}{|c|}{ Muscle respond } & \multirow{2}{*}{$\begin{array}{c}\text { Difference }^{\alpha} \\
(\mathrm{mm})\end{array}$} \\
\hline & Avg. & SD & Range & Avg. & $\mathrm{SD}$ & Range & Avg. & $\mathrm{SD}$ & Range & \\
\hline 1 & 3.13 & 0.25 & $2.78-3.67$ & 3.07 & 0.23 & $2.78-3.42$ & 3.95 & 1.21 & $2.73-5.16$ & $0.88^{b}$ \\
\hline 2 & 2.30 & 0.12 & $2.08-2.53$ & 2.10 & 0.21 & $1.84-2.43$ & 2.58 & 0.15 & $2.43-2.73$ & $0.48^{b}$ \\
\hline 3 & 3.73 & 0.61 & $2.48-4.81$ & 0.81 & 0.13 & $0.60-0.99$ & 5.49 & 1.02 & $4.47-6.50$ & $4.68^{b}$ \\
\hline 4 & 4.97 & 0.60 & $4.22-6.00$ & 0.90 & 0.09 & $0.745-1.04$ & 1.10 & 0.28 & $0.84-1.39$ & 0.20 \\
\hline 5 & 5.35 & 1.02 & $4.42-7.15$ & 0.79 & 0.16 & $0.546-0.943$ & 1.42 & 0.23 & $1.19-1.64$ & $0.63^{b}$ \\
\hline 6 & 7.95 & 0.75 & $6.70-8.93$ & 1.51 & 0.24 & $1.24-1.98$ & 2.41 & 0.52 & $1.89-2.93$ & $0.90^{b}$ \\
\hline 7 & 6.96 & 0.45 & $6.45-7.79$ & 4.33 & 0.26 & $4.02-4.66$ & 6.95 & 0.50 & $6.45-7.44$ & $2.62^{b}$ \\
\hline 8 & 1.98 & 0.19 & $1.71-2.18$ & 1.82 & 0.17 & $1.49-2.08$ & 2.98 & 0.08 & $2.90-3.16$ & $1.16^{b}$ \\
\hline 9 & 3.41 & 0.23 & $3.08-3.77$ & 2.83 & 0.26 & $2.48-3.42$ & 3.23 & 0.12 & $3.11-3.35$ & $0.40^{b}$ \\
\hline 10 & 2.74 & 0.28 & $2.33-3.03$ & 2.63 & 0.17 & $2.28-2.78$ & 2.93 & 0.15 & $2.78-3.08$ & 0.30 \\
\hline Overall avg. & 4.25 & 0.45 & $1.71-8.93$ & 2.08 & 0.19 & $0.60-4.66$ & 3.30 & 0.43 & $0.84-7.44$ & 1.23 \\
\hline
\end{tabular}

${ }^{a}$ Postfatigue minus baseline.

${ }^{b}$ Significant difference $(P<0.05)$.

pared with the baseline ( 8 of 10 being statistically significant). Seven of 10 subjects exhibited an increase in laxity from the first to second response trial after fatigue. The average anterior tibial translation (response test) after fatigue was $3.3 \mathrm{~mm}$ (range, 1.2 to 7.44 ). The average percentage increase in anterior tibial translation after fatigue was $32.5 \%$ (range, $11.4 \%$ to $85.2 \%$ ).

\section{Change in Average Muscle Reaction Time After Fatigue}

During baseline muscle testing before exercise, the spinal reflex was elicited $65 \%$ of the time in the medial quadri- ceps, $80 \%$ in the lateral quadriceps, and $100 \%$ in the gastrocnemius, lateral hamstring, and medial hamstring muscles (Table 3). Intermediate and voluntary responses were elicited consistently from all subjects in all muscle groups. However, both muscle reaction time and the number of responses significantly decreased after the muscles were fatigued.

Spinal Reflex. There was a statistically significant delay in muscle reaction time in the spinal reflex of the medial and lateral quadriceps muscles after fatigue (medial quadriceps, 60 to $80 \mathrm{msec}$; lateral quadriceps, 77 to $93 \mathrm{msec}$ ).

TABLE 3

Average Change in Muscle Reaction Time from Baseline to Postfatigue (in milliseconds)

\begin{tabular}{|c|c|c|c|c|c|c|c|c|c|}
\hline \multirow{3}{*}{ Category $^{\alpha}$} & \multicolumn{6}{|c|}{ Groups } & & & \\
\hline & \multicolumn{3}{|c|}{ Baseline } & \multicolumn{3}{|c|}{ Postfatigue } & \multicolumn{3}{|c|}{ Percentage muscle firing } \\
\hline & Avg. & Range & SEM & Avg. & Range & SEM & Baseline & Postfatigue & Change \\
\hline \multicolumn{10}{|l|}{ Spinal reflex } \\
\hline G & 54.2 & $(22-93)$ & 4.8 & 52.2 & $(30-67)$ & 4.3 & 100 & 55 & $-45^{b}$ \\
\hline LH & 75.9 & $(56-108)$ & 4.0 & 75.9 & $(53-92)$ & 4.4 & 100 & 70 & $-30^{b}$ \\
\hline MH & 69.3 & $(49-107)$ & 3.6 & 78.8 & $(45-104)$ & 4.3 & 100 & 65 & $-\mathbf{3 5}^{b}$ \\
\hline LQ & 76.9 & $(37-110)$ & 6.0 & $93.2^{c}$ & $(73-123)$ & 8.6 & 80 & 40 & $-40^{b}$ \\
\hline $\mathbf{M Q}$ & 59.6 & $(34-106)$ & 5.9 & $80.0^{c}$ & $(79-81)$ & 1.0 & 65 & 25 & $-40^{b}$ \\
\hline $\mathrm{PRO}^{d}$ & $\mathrm{G}, \mathrm{H}, \mathrm{Q}$ & & & $\mathrm{G}, \mathrm{H}$ & & & & & \\
\hline \multicolumn{10}{|c|}{ Intermediate response } \\
\hline $\mathbf{G}$ & 148.6 & $(128-171)$ & 6.7 & 157.5 & $(150-165)$ & 7.5 & 100 & 70 & $-30^{b}$ \\
\hline LH & 149.8 & $(133-172)$ & 3.9 & 156.0 & $(129-184)$ & 7.0 & 100 & 90 & -10 \\
\hline MH & 145.2 & $(134-161)$ & 2.7 & 159.1 & $(121-199)$ & 8.8 & 100 & 80 & -20 \\
\hline LQ & 145.2 & $(136-165)$ & 2.7 & $161.3^{c}$ & $(121-202)$ & 7.7 & 100 & 90 & -10 \\
\hline $\mathrm{MQ}$ & 143.5 & $(131-167)$ & 3.9 & $166.8^{c}$ & $(140-202)$ & 13.1 & 100 & 90 & -10 \\
\hline $\mathrm{PRO}^{d}$ & $\mathrm{Q}, \mathrm{H}, \mathrm{G}$ & & & $\mathrm{Q}, \mathrm{H}, \mathrm{G}$ & & & & & \\
\hline \multicolumn{10}{|c|}{ Voluntary response } \\
\hline G & 272.9 & $(230-316)$ & 12.3 & 304.3 & $(257-392)$ & 43.9 & 100 & 50 & $-50^{b}$ \\
\hline LH & 249.3 & $(186-367)$ & 15.7 & 265.9 & $(221-373)$ & 15.2 & 100 & 100 & 0 \\
\hline MH & 237.9 & $(196-283)$ & 8.4 & $274.9^{c}$ & $(218-353)$ & 14.3 & 100 & 100 & 0 \\
\hline $\mathbf{L Q}$ & 237.6 & $(186-332)$ & 13.4 & $310.8^{c}$ & $(241-402)$ & 25.2 & 100 & 100 & 0 \\
\hline $\mathbf{M Q}$ & 243.0 & $(173-318)$ & 14.4 & $325.0^{c}$ & $(241-402)$ & 37.2 & 100 & 100 & 0 \\
\hline $\mathrm{PRO}^{d}$ & $\mathrm{Q}, \mathrm{H}$ & & & $\mathrm{Q}, \mathrm{H}$ & & & & & \\
\hline
\end{tabular}

${ }^{\alpha} \mathrm{G}$, gastrocnemius; LH, lateral hamstring; $\mathrm{MH}$, medial hamstring; LQ, lateral quadriceps; MQ, medial quadriceps.

${ }^{b}$ Significant decrease in number of responses from baseline to postfatigue.

c Significant change in response time $(P \leq 0.05)$, adjusted for multiple comparisons.

${ }^{d}$ PRO, predominant recruitment order. 
All muscle groups tested also showed a significant decrease in the number of responses from baseline to fatigue. The percentage of muscle firing in both the medial and lateral quadriceps muscles decreased $40 \%$ after exercise to fatigue, and the firing rates of the hamstring muscles similarly decreased (lateral hamstring, 30\%; medial hamstring, $35 \%$ ). Interestingly, the muscle firing rate of the gastrocnemius muscle decreased $45 \%$ after fatigue, even though that muscle group was not specifically exercised on the isokinetic dynamometer. The predominant muscle recruitment order did not change from baseline to postfatigue (gastrocnemius, hamstring, quadriceps).

Intermediate Response. There was a significant delay in muscle reaction time in the intermediate response after fatigue for the medial and lateral quadriceps muscles. There was also a significant decrease $(30 \%)$ in the number of intermediate responses after fatigue in the gastrocnemius muscle. The predominant muscle recruitment order after muscle fatigue remained unchanged from the baseline (quadriceps, hamstring, gastrocnemius).

Voluntary Response. There were delays in muscle reaction time in the voluntary response to anterior tibial translation after fatigue for all muscle groups tested (three were significant-medial hamstring, lateral quadriceps, and medial quadriceps). Delay averaged $17.5 \%$ in the gastrocnemius muscle, $13.5 \%$ in the hamstring muscle, and $28.5 \%$ in the quadriceps muscle. Again, there was a significant decrease $(50 \%)$ in the number of responses after fatigue in the gastrocnemius muscle. The predominant muscle recruitment order after fatigue did not change (quadriceps, hamstring, gastrocnemius).

\section{Relationship of Anterior Tibial Translation Increase and Delay in Muscle Reaction Time}

Spinal Reflex. No correlation was found between the change in anterior tibial translation and the spinal reflex of any of the five muscle groups.

Intermediate Response. The change in anterior tibial translation strongly correlated with a delayed intermediate response time in the medial hamstring $(P=0.02)$ and the quadriceps muscles $(P=0.035)$. The corresponding correlations in the gastrocnemius and lateral hamstring muscles were not significant. Interestingly, delayed intermediate response time in the quadriceps muscle strongly correlated $(P<0.001)$ with the percentage of quadriceps muscle fatigue.

Voluntary Response. The change in anterior tibial translation strongly correlated with delayed voluntary response time in the lateral hamstring $(P=0.02)$, quadriceps $(P=$ $0.036)$, and gastrocnemius muscles $(P=0.045)$. The corresponding correlation in the medial hamstring was not significant.

\section{Strength and Activity Level}

Strength (peak torque [in foot-pounds]/body weight [in pounds]) of both the quadriceps and hamstring muscles correlated with the activity levels of the participants (quadriceps muscle, $r=0.83$; hamstring muscle, $r=0.90$ ) (Fig. 1).

\section{Men Versus Women}

There were no significant differences between men and women in terms of strength normalized to body weight, percentage of fatigue, muscle reaction time, or anterior tibial translation.

\section{DISCUSSION}

Previous work by Pope et al. ${ }^{14}$ on the concept of a muscle protection response at the knee joint indicated that voluntary muscle activity was too slow to prevent joint injury. In fact, recent work by Yasuda et al. ${ }^{26}$ documents ACL failure as quickly as 35 to $70 \mathrm{msec}$ after the application of a damaging valgus force.

In this study, baseline testing demonstrated spinal cord level responses occurring between 22 and $110 \mathrm{msec}$ after
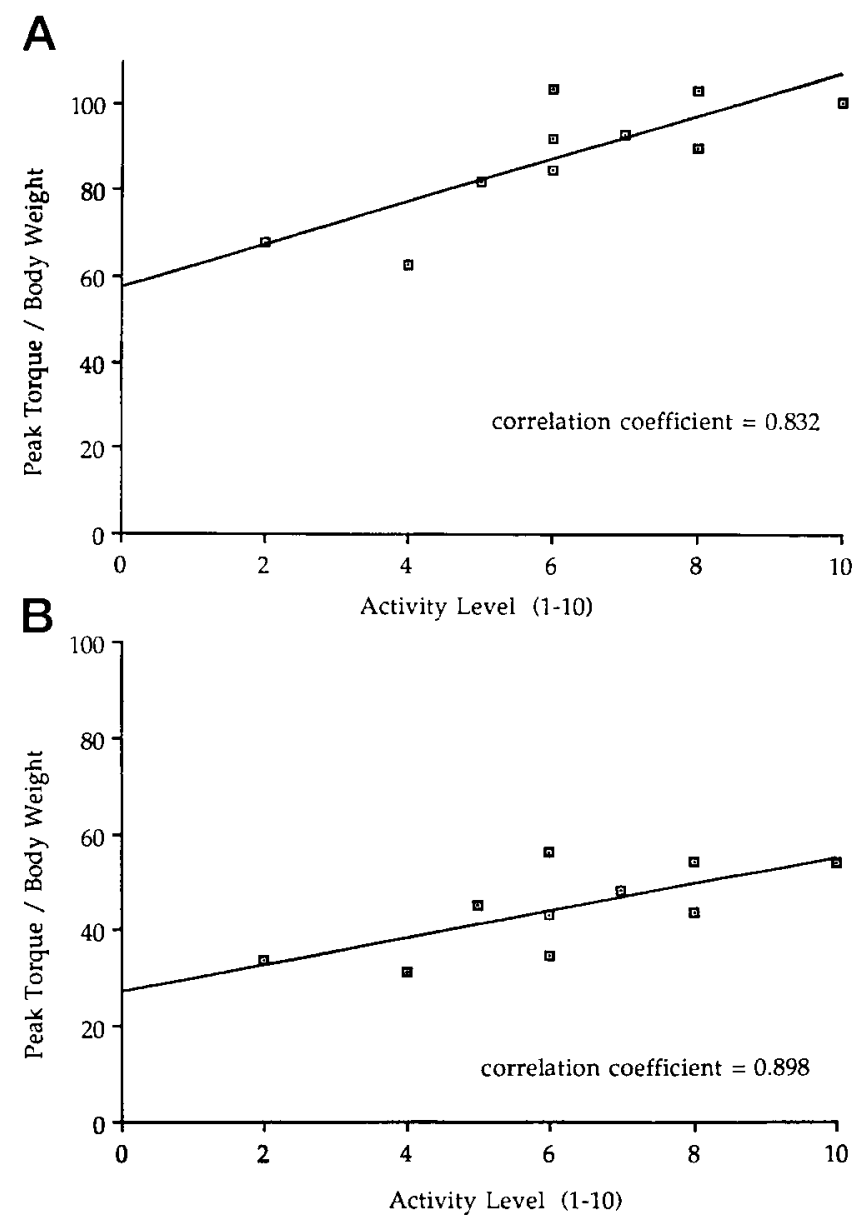

Figure 1. Strength (peak torque [in foot-pounds]/body weight [in pounds]) of both the quadriceps and hamstring muscles correlated with the baseline activity levels of the participants. A, activity level versus quadriceps muscle strength. $B$, activity level versus hamstring muscle strength. 
the induced anterior tibial translation. Therefore, the possibility of spinal cord level protection against injury should not be ruled out. Depending on the individual muscle (gastrocnemius, lateral hamstring, medial hamstring, lateral quadriceps, or medial quadriceps), the spinal reflex was detected in $65 \%$ to $100 \%$ of the trials before fatigue and less frequently after exercise (Table 3 ). The significance of not always detecting a spinal reflex is unclear at this time. However, localized muscle fatigue is not expected to increase spinal reflex latency even though a delay in muscle reaction time is anticipated. ${ }^{6}$

The intermediate response represents activity at the spinal cord level but depends on supraspinal input. ${ }^{5}$ Therefore, in this investigation, it was classified as cortical-level activity. Anticipation of potentially damaging forces would probably be needed to make this level of control a factor in knee injury prevention because of the time span to muscle activation involved. In the intermediate response segment of the EMG recording after fatigue was induced, the muscle reaction time did increase in the gastrocnemius, hamstring, and quadriceps muscles. Additionally, all voluntary muscle reaction times after fatigue showed increases in duration. These findings are in agreement with those reported by Sheerer and Berger, ${ }^{15}$ which indicate that as little as a $15 \%$ decrease in maximum strength can cause a reduction in muscle reaction time. These findings are also in agreement with both Woods et al. ${ }^{25}$ and Bigland-Ritchie et al., ${ }^{2}$ who reported a decrease in motoneuron firing rates during muscle fatigue in the upper extremity; the reason for this outcome was thought to be that as motoneuron firing rates decrease, muscle reaction time should lengthen. The findings in this study do not agree with those of Greig et al. ${ }^{10}$ who reported an increase in the EMG activity-to-muscle force ratio after fatigue. In this study, EMG activity (especially at the spinal cord level) appeared to decrease with fatigue.

Although the changes in anterior tibial translation after muscle fatigue reported in this study are relatively small (range, 0.2 to $4.7 \mathrm{~mm}$ ), they appear to be real in light of our previously published reproducibility data. ${ }^{21}$ However, we continue to view these changes with caution because the significance of this level of change in anterior tibial translation is not known in terms of knee joint kinematics in the short or long term. The increases in anterior tibial translation in most participants reported in this study exceed the increases Steiner et al., ${ }^{17}$ who used a knee arthrometer to measure anterior tibial translation after sports activity in an athletic population, reported (average increase of $0.5 \mathrm{~mm} ; 18 \%$ to $20 \%$ ) after either 90 minutes of basketball practice or a $10-\mathrm{km}$ race. Grana and Muse ${ }^{9}$ reported very similar data in a group of healthy controls who exhibited a $21 \%$ increase in anterior tibial translation $(0.1$ to $0.9 \mathrm{~mm}$ ) after 20 minutes on a bicycle ergometer at $60 \%$ of $\dot{\mathrm{VO}}_{2} \max$ for untrained persons ages 20 to 39 years. Similarly, Stoller et al. ${ }^{18}$ reported a $14 \%$ increase in rotational laxity (in millimeters) of the tibia at $90^{\circ}$ of knee flexion using a $10 \mathrm{~N}$-m load after a 30 -minute, 3.5-mile run.

Currently, the exact source and distribution of the increased anterior tibial translation in this study is unclear, but a combination of sources is most likely, namely, vis- coelastic changes in the collagenous tissues of the knee and fatigued muscle stabilizers. Cyclic loading of tendons and ligaments during exercise can account for up to a $5 \%$ increase in tendon and ligament length. ${ }^{1,23}$ This can, in part, be due to an increase in temperature, which was not monitored in this study. ${ }^{12,24}$ Because the average ACL length has been reported at $38 \mathrm{~mm},{ }^{7}$ a $5 \%$ strain would be expected to produce an additional $1.9 \mathrm{~mm}$ in ACL length. However, this rate of strain could not account for the $11.4 \%$ to $85.2 \%$ increase in anterior tibial translation reported here. Also, with this slight increase in length, cyclically loaded collagenous tissues can be expected to show a proportional decrease in stiffness, causing a reduction in ligament strength to failure. ${ }^{20}$

Interestingly, this increase in anterior tibial translation (11.4\% to $85.2 \%$ ) may best be explained by the fatiguing of the active knee stabilizers: the quadriceps and hamstring muscles. Keep in mind that Bongiovanni and Hagbarth ${ }^{3}$ have shown that intrafusal muscle fiber fatigue leads to a reduction in the voluntary drive conveyed to the alphamotoneurons via the gamma loop. With this decrease in voluntary drive, a decrease in the force of hamstring, quadriceps, and gastrocnemius muscle contractions across the knee would be expected. Markolf et al. ${ }^{13}$ predicted a $1-\mathrm{mm}$ increase in anteroposterior translation of the tibia with each $100 \mathrm{~N}$ decrease in joint compressive load. Their in vitro work suggested that if the peak torque of knee stabilizing muscles decreases, knee joint laxity would increase.

Muscles-relaxed anterior tibial translation tests after muscle fatigue would have helped answer the question concerning the origin of the increase in anterior tibial translation. Unfortunately, true EMG-monitored muscle relaxation was not possible; several subjects could not fully relax their lower extremities after their exercise periods because the workouts were vigorous.

Currently, efforts continue to determine which parameters in which muscles at what neural control level are the most important for dynamic knee joint stability. Various conditioning programs have been tested to see if these muscle reaction parameters can be altered. ${ }^{22}$

\section{CONCLUSIONS}

1. Quadriceps and hamstring isokinetic muscle exercise to the point of muscle fatigue $(50 \%)$ can increase anterior tibial translation at the knee.

2. The viscoelastic properties of the collagenous tissues supporting the knee cannot alone account for the increases in anterior tibial translation seen after exercise. The increases in anterior tibial translation appear to be due, in part, to fatiguing the quadriceps and hamstring muscles.

3. Hamstring and quadriceps muscle fatigue does not appear to change the order of muscle recruitment in response to anterior tibial translation. However, muscle reaction time is slowed after fatigue.

4. The null hypothesis of this investigation was rejected because fatigue does appear to change the muscle reaction time and anterior tibial translation parameters at the knee. 


\section{REFERENCES}

1. Abrahams M: Mechanical behaviour of tendon in vitro. Med Biol Eng 5: 433-443, 1967

2. Bigland-Ritchie BR, Dawson NJ, Johansson RS, et al: Reflex origin for the slowing of motoneuron firing rates in fatigue of human voluntary contractions. J Physiol 379: 451-459, 1986

3. Bongiovanni LG, Hagbarth KE: Tonic vibration reflexes elicited during fatigue from maximal voluntary contractions in man. J Physiol (Lond) 423: $1-14,1990$

4. Dunn MG, Silver FH: Viscoelastic behavior of human connective tissues: Relative contribution of viscous and elastic components. Connect Tissue Res 12: 59-70, 1983

5. Eccles JC: Evolution of the brain: Creation of the self, in Evolution of the Brain. New York, Routledge, 1989, pp 61-67

6. Eke-Okoro ST: The H-reflex studied in the presence of alcohol, aspirin, caffeine, force and fatigue. Electromyogr Clin Neurophysiol 22: 579-589, 1982

7. Girgis FG, Marshall JL, Al Monajem ARS: The cruciate ligaments of the knee joint: Anatomical, functional and experimental analysis. Clin Orthop 106: 216-231, 1975

8. Goldfuss AJ, Morehouse CA, LeVeau BF: Effect of muscular tension on knee stability. Med Sci Sports 5: 267-271, 1973

9. Grana WA, Muse G: The effect of exercise on laxity in the anterior cruciate ligament deficient knee. Am J Sports Med 16: 586-588, 1988

10. Greig G, Hortobagyi T, Sargeant AJ: Quadriceps surface EMG and fatigue during maximal dynamic exercise in man. J Physiol 369: 180P, 1985

11. Kowalk DL, Wojtys EM, Disher $\mathrm{J}$, et al: Quantitative analysis of the measuring capabilities of the KT-1000 knee ligament arthrometer. Am J Sports Med 21: 744-747, 1993

12. Lam TC, Thomas CG, Shrive NG, et as: The effects of temperature on the viscoelastic properties of the rabbit medial collateral ligament. J Biomech Eng 112: 147-152, 1990
13. Markolf $K L$, Graff-Radford A, Amstutz HC: In vivo knee stability. A quantitative assessment using an instrumented clinical testing apparatus. $J$ Bone Joint Surg 60A: 664-674, 1978

14. Pope MH, Johnson RJ, Brown DW, et al: The role of the musculature in injuries to the medial collateral ligament. J Bone Joint Surg 61A:398 -402, 1979

15. Sheerer N, Berger RA: Effects of various levels of fatigue on reaction time and movement time. Am Correct Ther J 26: 146-147, 1972

16. Skinner HB, Wyatt MP, Stone ML, et al: Exercise-related knee joint laxity. Am J Sports Med 14: 30-34, 1986

17. Steiner ME, Grana WA, Chillag $\mathrm{K}$, et al: The effect of exercise on anteriorposterior knee laxity. Am J Sports Med 14: 24-29, 1986

18. Stoller DW, Markolf $\mathrm{KL}$, Zager SA, et al: The effects of exercise, ice, and ultrasonography on torsional laxity of the knee. Clin Orthop 174:172-180, 1983

19. Wang CJ, Walker PS: Rotatory laxity of the human knee joint. J Bone Joint Surg 56A: 161-170, 1974

20. Weisman $\mathrm{G}$, Pope $\mathrm{MH}$, Johnson RJ: Cyclic loading in knee ligament injuries. Am J Sports Med 8: 24-30, 1980

21. Wojtys EM, Huston LJ: Neuromuscular performance in normal and anterior cruciate ligament-deficient lower extremities. Am J Sports Med 22: 89-104, 1994

22. Wojtys EM, Huston LJ, Taylor PD et al: Neuromuscular adaptations in isokinetic, isotonic, and agility training programs. Am J Sports Med 24: 187-192, 1996

23. Woo SL: Mechanical properties of tendons and ligaments: I. Quasi-static and nonlinear viscoelastic properties. Biorheology 19: 385-396, 1982

24. Woo SL, Lee TQ, Gomez MA, et al: Temperature dependent behavior of the canine medial collateral ligament. J Biomech Eng 109: 68-71, 1987

25. Woods JJ, Furbush F, Bigland-Ritchie B: Evidence for a fatigue-induced reflex inhibition of motoneuron firing rates. I Neurophysiol 58: 125-137, 1987

26. Yasuda K, Erickson AR, Johnson RJ, et al: Dynamic strain behavior in the medial collateral and anterior cruciate ligaments during lateral impact loading. Trans Orthop Res Soc 17: 127, 1992 